\title{
The mechanism of mid-latitude $\mathrm{Pi} 2$ waves in the upper ionosphere as revealed by combined Doppler and magnetometer observations
}

\author{
V. A. Pilipenko ${ }^{1,2}$, E. N. Fedorov ${ }^{3}$, M. Teramoto ${ }^{2}$, and K. Yumoto ${ }^{4}$ \\ ${ }^{1}$ Space Research Institute, Moscow, Russian Federation \\ ${ }^{2}$ Solar-Terrestrial Environment Laboratory, Nagoya University, Japan \\ ${ }^{3}$ Institute of Physics of the Earth, Moscow, Russian Federation \\ ${ }^{4}$ International Center for Space Weather Science and Education, Kyushu University, Fukuoka, Japan
}

Correspondence to: V. A. Pilipenko (pilipenk@augsburg.edu)

Received: 23 November 2012 - Revised: 19 March 2013 - Accepted: 21 March 2013 - Published: 17 April 2013

\begin{abstract}
The interpretation of simultaneous ionospheric Doppler sounding and ground magnetometer observations of low-latitude Pi2 waves is revised. We compare the theoretical estimates of the ionospheric Doppler velocity for the same amplitude of the ground magnetic disturbances produced by a large-scale compressional mode and an Alfvén mode. The plasma vertical displacement caused by the wave electric field is shown to be the dominating effect. Taking into account the correction of the previous paper, the observations of low-latitude Pi2 in the F layer ionosphere by Doppler sounding and SuperDARN (Super Dual Auroral Radar Network) radars give consistent results. We suggest that the Doppler response to Pi2 waves is produced by the Alfvén wave component, but not the fast-mode component, whereas the ground magnetic signal is composed from both Alfvén and fast magnetosonic modes.
\end{abstract}

Keywords. Magnetospheric physics (MHD waves and instabilities)

\section{Introduction: simultaneous Doppler and magnetome- ter ULF observations}

Doppler sounding is one of the few experimental facilities to detect in situ the interaction of magnetospheric ultra-lowfrequency (ULF) disturbances with the ionosphere. The ionospheric response to various periodic and transient disturbances in the ULF range Pc5 (Reddy et al., 1994), Pi2 (Alperovich et al., 1991; Menk, 1992; Wright at al., 1997), Pc34 (Sutcliffe and Poole, 1984; Menk et al., 2007), and storm sudden commencement (SC) (Yumoto et al., 2009) as mea- sured by the Doppler technique have been reported. These observations were interpreted by considering either Alfvén transverse mode or fast compressional mode.

Special attention was devoted to the Doppler studies of Pi2 pulsations (typical periods $\sim 1-2 \mathrm{~min}$ ). These transient (duration of $\sim$ a few periods) signals are widely used as a global wave marker of the substorm onset (e.g., see review by Keiling and Takahashi, 2011) or bursty/impulsive processes in the magnetotail (Shiokawa et al., 1998). Therefore, a mechanism of $\mathrm{Pi} 2$ propagation from the nightside magnetotail to low-latitude ground stations is important to know.

From observations of the mid-latitude bottom-side F layer disturbed by Pi2 magnetic pulsations using vertical HF (highfrequency) soundings, Grant and Cole (1992) found that the amplitude of the observed Doppler velocity was constant with height and was predominantly due to the vertical motion driven by an E-W Pi2 pulsation electric field. On the other hand, Marshall and Menk (1999) reported that the Doppler frequency variations caused by low-latitude nighttime Pi2 and irregular Pc4 were proportional to their frequency. This proportionality was interpreted as the indication that the plasma compression was dominant for the ionospheric response to low-latitude Pi2.

Multipoint Doppler sounding at the mid-latitude observatory Budkov (Chum et al., 2009) revealed simultaneously at several radiopaths Pi2 pulsations, well correlated with geomagnetic signatures. The estimated time/phase shifts between geomagnetic and Doppler signals changed from case to case, lag mostly being from $80^{\circ}$ to $180^{\circ}$.

Ikeda et al. (2009) analyzed in detail the simultaneous observations by HF radar and flux-gate magnetometer located 
at the mid-latitude station Paratunka. The detected variations in the F layer and on the ground revealed high coherence (>0.6) for half of the $114 \mathrm{Pi} 2$ events. Among these highcoherent events, the phase delay between magnetic and ionospheric variations at the dominant frequency was $84^{\circ} \pm 27^{\circ}$ during the midnight sector (21:00-03:00 LT), but not in other LT sectors. The ratio between the Doppler velocity and ground magnetic disturbance did not depend on the Pi2 frequency. They interpreted their observational results as a manifestation of the cavity mode nature of nighttime $\mathrm{Pi} 2$ pulsations at low latitudes.

Commonly, $\mathrm{Pi} 2$ pulsations at low latitudes have been interpreted as a signature of the plasmaspheric cavity mode, formed by fast magnetosonic waves trapped within the nighttime plasmasphere (Yeoman and Orr, 1989). The Earth, despite its relatively small scale as compared with the fastmode wavelength, is considered to be a good reflector of incident waves, resulting in the formation of a resonant wave cavity within the plasmasphere (Nose, 2010). Numerous ground-satellite studies examined characteristics of Pi2 pulsations in the magnetosphere. Using the equatorial orbiting AMPTE/CCE satellite, Takahashi et al. (1995) found that radial and compressional magnetic components of Pi2 pulsations in the magnetosphere at $L<5$ had a high coherence with the North-South $(\mathrm{N}-\mathrm{S})$ component at the lowlatitude station Kakioka $(L=1.2)$ on the nightside. Identifying the location of the plasmapause in the electron density data from the CRRES satellite, Takahashi et al. (2003) confirmed that magnetospheric compressional $\mathrm{Pi} 2$ pulsations were indeed confined within the plasmapause. According to the box model of the cavity mode in the plasmasphere (Takahashi et al., 2001), the phase delay between transverse electric and compressional magnetic components is to be $-90^{\circ}$ near the inner boundary, and this structure was supposed to be transmitted to the lower ionosphere.

Observations at low-altitude $(\sim 800-1000 \mathrm{~km})$ satellites have also shown that the dominant component of the $\mathrm{Pi} 2$ wave structure in the upper ionosphere is the compressional magnetic component (Sutcliffe and Lühr, 2003; Han et al., 2004).

However, the dominance of the fast-mode contribution into the ULF wave structure in the magnetosphere does not necessarily indicate that the same would be valid inside the ionosphere. Indeed, upon approaching the Earth, a fast magnetosonic wave is to be reflected from a region with high Alfvén velocities, so it can penetrate through the ionosphere to the ground as an evanescent mode only.

Moreover, in a realistic inhomogeneous magnetospheric plasma immersed into curved magnetic field, magnetohydrodynamic (MHD) modes are to be coupled, so even a cavitylike Pi2 wave is to comprise some contribution of Alfvénic disturbances. Alfvén waves have no reflection point, so they can reach the bottom ionosphere directly. Thus, any comprehensive theory of Pi2 impact on the ionosphere should include both Alfvén and fast compressional modes into con- sideration. Upon transmission through the ionosphere both these modes with a large scale in the azimuthal direction provide a main magnetic response on the ground in the same $\mathrm{N}-\mathrm{S}$ component. Thus, ground magnetometer observations only cannot resolve the physical mechanism of ULF waves.

The basic model for the interpretation of the ionospheric Doppler effects was developed by Poole et al. (1988) and Sutcliffe and Poole (1990) on the basis of numerical solution of the Alfvén wave transmission through the ionosphere and numerical integration over the radio wave propagation path in a wave-disturbed ionosphere. However, this numerical model does not provide an easy-to-use analytical formula for the estimate of expected effect under various ionospheric and ULF wave parameters. Moreover, this model considered an incident wave an Alfvén mode only. An analytical model to interpret the deviations of the sounding radio wave frequency caused by MHD disturbances was proposed by Pilipenko et al. (2010). In this model, the variations of Doppler velocity induced by compressional and Alfvén waves were theoretically estimated on the basis of the thin ionosphere approximation.

In this paper we consider possible physical mechanisms of the Doppler response to the magnetospheric $\mathrm{Pi} 2$ wave. Here we present simple analytical relations which describe with reasonable accuracy the Doppler response to both the compressional and Alfvén modes. The presented results are the extension of our previous analytical model, earlier applied to interpret the simultaneous Doppler and magnetic signatures during SC (Pilipenko et al., 2010). On the basis of these theoretical predictions, we re-examine the interpretation of the simultaneous ionospheric and magnetic observations at low latitudes made by Ikeda et al. (2010).

\section{Ionospheric Doppler response to magnetospheric MHD waves}

\subsection{Basic relationships}

For a near-vertical sounding, the frequency $f_{\mathrm{R}}$ deviation of a radio wave, propagating through the ionospheric plasma disturbed by ULF wave fields, can be derived via the effective Doppler velocity $V^{*}$ as follows (Poole et al., 1988):

$$
\frac{\Delta f_{\mathrm{R}}}{f_{\mathrm{R}}}=-\frac{2 V^{*}}{c}, \quad V^{*}=\frac{d}{d t} \int_{0}^{Z_{\mathrm{R}}} \mu d z .
$$

Here $\mu\left(f_{\mathrm{R}}, \boldsymbol{B}(z, t), N(z, t)\right)$ is the real part of the radio wave refractive index, and $N$ denotes the electron concentration. The total magnetic field $\boldsymbol{B}(z, t)=\boldsymbol{B}_{0}+\boldsymbol{B}(z, t)$ is a sum of the geomagnetic field $\boldsymbol{B}_{0}$ and the disturbance $\boldsymbol{B}$, composed of the transverse $\boldsymbol{B}_{\perp}$ and field aligned $\boldsymbol{B}_{\|}$components. The geomagnetic field $\boldsymbol{B}_{0}=\{H, 0, Z\}$ is inclined by the angle $I$ to the ground surface ( $I>0$ in the Northern Hemisphere). The angle between $\boldsymbol{B}_{0}$ and the radio wave vector is $\pi / 2-I$. 
In the coordinate system chosen the axis $x$ is directed southward, $z$ upward, and $y$ eastward. The integration in Eq. (1) is to be performed along $z$ from the bottom of the ionosphere $(z=0)$ up to the reflection point $z_{\mathrm{R}}\left(\right.$ where $\left.\mu\left(z_{\mathrm{R}}\right)=0\right)$ at altitude $z_{h}=z_{\mathrm{R}}+h$.

According to Sutcliffe and Poole (1989, 1990), the total Doppler effect $V^{*}$ can be presented as the sum of several effects:

$V^{*}=V_{1}+V_{2}+V_{3}$.

The term $V_{1}$ in Eq. (2), caused by the variations of the refractive index due to magnetic field fluctuations, is commonly small as compared with other terms and will not be considered here.

The term $V_{2}$ in Eq. (2) is related to the plasma convection with velocity $\boldsymbol{V}$ caused by the wave electric field $\boldsymbol{E}=\left\{E_{\mathrm{x}}, E_{\mathrm{y}}, E_{\mathrm{z}}\right\}$. In a highly conductive ionosphere the field-aligned component of the electric field vanishes: $E_{\|}=$ $-E_{\mathrm{X}} \cos I-E_{\mathrm{Z}} \sin I=0$. The vertical plasma drift velocity $V_{\mathrm{z}}$ caused by the East-West (E-W) component of the wave electric field $E_{\mathrm{y}}$ is as follows:

$V_{\mathrm{z}}=\frac{E_{\mathrm{y}} \cos I}{B_{0}}$.

Thus, in the laterally homogeneous ionosphere the wave electric field produces the Doppler effect

$V_{2}=-\frac{\cos I}{B_{0}} \int_{0}^{Z_{\mathrm{R}}} E_{\mathrm{y}} \frac{\partial \mu}{\partial z} d z$

The term $V_{3}$ in Eq. (2) is caused by the plasma compression produced by the field-aligned magnetic component $B_{\|}$:

$V_{3}=-\int_{0}^{Z_{\mathrm{R}}} \frac{\partial \mu}{\partial N} N(\nabla \cdot \boldsymbol{V}) d z=\int_{0}^{Z_{\mathrm{R}}} \frac{\partial \mu}{\partial N} \frac{N}{B} 0 \frac{\partial B_{\|}}{\partial t} d z$.

For analytical estimates we approximate the vertical profile of the ionospheric plasma $N(z)$ below the peak of the $\mathrm{F}$ layer with the exponential function with the scale $a$, such that

$\frac{1}{N(z)} \frac{\partial N(z)}{\partial z}=a^{-1}=$ const.

The main contribution into the height-integrated Doppler effect (Eqs. 4 and 5) is produced by a relatively small region near the radio wave reflection altitude $z \simeq z_{\mathrm{R}}$, so the local approximation Eq. (6) seems reasonable.

We compare the Doppler velocities caused by different MHD wave modes for the same magnitude of the ground magnetic disturbance, corresponding to the $\mathrm{N}-\mathrm{S} H$ component, that is, $B_{\mathrm{x}}^{(\mathrm{g})}=-H$. In the estimates below we use the analytical expressions for the reflection and transmission coefficients of plane harmonics of MHD waves $\propto \exp (-i \omega t+$ $i \boldsymbol{k r}$ ) (where $\omega$ and $\boldsymbol{k}$ are the wave angular frequency and wave vector) obtained within the thin sheet approximation of the ionosphere (Alperovich and Fedorov, 2007). This sheet has the anisotropic height-integrated Pedersen and Hall conductances, $\Sigma_{\mathrm{P}}$ and $\Sigma_{\mathrm{H}}$, and is situated at the altitude $h$ above the ground. The plasma of the upper ionosphere is characterized by the Alfvén wave conductance $\Sigma_{\mathrm{A}}$ related to Alfvén velocity $V_{\mathrm{A}}$ with $\Sigma_{\mathrm{A}}=\left(\mu_{0} V_{\mathrm{A}}\right)^{-1}$, and by an Alfvén wave number $k_{\mathrm{A}}=\omega / V_{\mathrm{A}}$ (Nishida, 1978).

The wave field $\boldsymbol{B}$ above the ionospheric layer is composed of incident $\boldsymbol{B}^{(i)}$ and reflected $\boldsymbol{B}^{(r)}$ waves of partial Alfvén and fast compressional modes. The experimentally measurable quantity is a total field $\boldsymbol{B}=\boldsymbol{B}^{(i)}+\boldsymbol{B}^{(r)}$; hence, all the relationships below are given for the total field. Using the basic formulas from Alperovich and Fedorov (2007), which relate amplitudes of waves incident and reflected from the ionosphere, as well as transmitted through the ionosphere to the ground, the transformation coefficients from incident Alfvén and fast waves into the magnetic (or transverse-electric TE) mode in the atmosphere can be derived. The ground response, when the conductivity and displacement currents in the atmosphere are neglected, is due to this mode only. The ULF field variations in the $\mathrm{E}-\mathrm{W}$ direction are assumed to be very large as compared with the $\mathrm{N}-\mathrm{S}$ direction $\left(k_{\mathrm{y}} / k_{\mathrm{x}} \gg 1\right)$. This approximation holds well for low-latitude Pi2 disturbances (Sutcliffe and Yumoto, 1991). Simple analytical relationships can be obtained in the asymptotic case $k_{\mathrm{y}} \rightarrow 0$. The ground conductivity is assumed to be high, i.e. $k \delta_{\mathrm{g}} \ll 1$, where $\delta_{\mathrm{g}}$ is the skin depth in the ground.

The effective tool for the discrimination of ULF modes interacting with the system magnetosphere-thin-ionosphereatmosphere-ground is the apparent admittance

$X=\frac{B^{(\mathrm{g})}}{\mu_{o} E} \quad X[\mathrm{~S}] \simeq 0.8 \frac{B^{(g)}[\mathrm{nT}]}{E\left[\mathrm{mV} \mathrm{m}^{-1}\right]}$

where $B^{(\mathrm{g})}$ and $E$ are relevant ground magnetic and ionospheric electric fields and $\mu_{o}$ is the magnetic constant. Below we will demonstrate that the admittances of different MHD modes are very different.

\subsection{Fast compressional mode}

Azimuthally large-scale fast mode, or fast magnetosonic (FMS) mode, is described by the dispersion relationship $k_{\mathrm{A}}(z)^{2}=k_{\mathrm{x}}^{2}+k_{\mathrm{z}}^{2}$. In a non-propagating regime, when $k_{\mathrm{x}}^{2}<0$, a low-frequency compressional mode cannot reach directly the E layer, and it reflects at some height above the ionosphere. So, in the ionosphere this mode happens to be in a tunneling (evanescent) regime.

For the large-scale wave structure $(k h \ll 1, k z \ll 1)$, magnetic components do not vary noticeably along the altitude. The relationship between the compressional wave and its ground response is (Pilipenko et al., 2010)

$\frac{B_{\|}}{B_{\mathrm{x}}^{(\mathrm{g})}}=-(1-i p) \cos I, \quad \frac{B_{\perp}}{B_{\mathrm{x}}^{(\mathrm{g})}}=-(1-i p) \sin I$. 
Here we have introduced the coefficient $p=\omega \mu_{0} \tilde{\Sigma}_{\mathrm{C}} h$ characterizing the transparency of the ionosphere to an incident compressional mode. The Cowling-like combination of height-integrated conductivities is $\Sigma_{\mathrm{C}}=\Sigma_{\mathrm{P}}+\Sigma_{\mathrm{H}}^{2} / \Sigma_{\mathrm{P}}$ (Schunk and Nagy, 2009), whereas $\tilde{\Sigma}_{\mathrm{C}}=\Sigma_{\mathrm{P}}+\Sigma_{\mathrm{H}}^{2} /\left(\Sigma_{\mathrm{P}}+\right.$ $\left.\Sigma_{\mathrm{A}}|\sin I|\right)$ is the modified Cowling conductance. At dayside, where $\Sigma_{\mathrm{P}} \ll \Sigma_{\mathrm{A}}$, the difference between them is insignificant, $\tilde{\Sigma}_{\mathrm{C}} \simeq \Sigma_{\mathrm{C}}$.

During daytime, when $|p| \simeq 1$, the ionosphere partly absorbs the downgoing fast mode. At nightside, when $|p| \ll 1$, the fast mode practically does not "feel" the ionosphere.

In contrast to magnetic components, the wave electric field $E(z)$ does vary essentially with altitude. Below $\mathrm{F}$ layer peak and for the nighttime conditions $(|p| \ll 1)$ the azimuthal electric field of fast mode $E_{\mathrm{y}}(z)$ is related to the ground magnetic response as

$$
E_{\mathrm{y}}(z)=-i \omega z_{\mathrm{h}} B_{\mathrm{x}}^{(\mathrm{g})} .
$$

A simple analytical estimate of $V_{2}$ can be obtained taking into account that the integrand in Eq. (4) decays fast away from the reflection point; hence the integral value is determined by the integrand behavior in the vicinity of the reflection point. Using this approximation, Pilipenko et al. (2010) obtained

$V_{2}=-i \omega z_{\mathrm{h}} \frac{\cos I}{B_{0}} B_{\mathrm{x}}^{(\mathrm{g})}$.

This relationship predicts that for quasi-periodic disturbance $\propto \exp (-i \omega t)$ the variations of Doppler velocity and magnetic field above a perfectly conductive ground are to be inquadrature $\left(-90^{\circ}\right.$ phase shift).

The apparent admittance of the multi-layer magnetosphere-ionosphere-atmosphere-ground system to a fast mode is to be

$X^{(\mathrm{F})}=\frac{B_{\mathrm{x}}^{(\mathrm{g})}}{\mu_{0} E_{\mathrm{y}}}=-\left(i \omega \mu_{0} z_{\mathrm{h}}\right)^{-1}$.

The absolute value of the fast-mode admittance can be estimated from approximate relation $\left|X^{(\mathrm{F})}\right|[\mathrm{S}] \simeq$ $127 T[\mathrm{~s}] / z_{\mathrm{h}}[\mathrm{km}]$.

The simple analytical estimate of the plasma compression mechanism $V_{3}$ can be obtained in a similar way. However, the contribution of $V_{3}$ mechanism as compared to $V_{2}$ turns out to be small: $\left|V_{3} / V_{2}\right| \simeq a / z_{\mathrm{R}}$.

\subsection{Alfvén mode}

Alfvén shear waves are MHD waves carrying oscillatory field-aligned electric current along magnetic field lines without geometric attenuation. These waves have no turning point and can easily reach the lower ionosphere. For the Alfvén wave with infinitely long azimuthal scale $\left(k_{\mathrm{y}} \rightarrow 0\right)$, the Doppler effect caused by the vertical electric drift vanishes as $E_{\mathrm{y}} \rightarrow 0$. However, an incident Alfvén wave upon the interaction with an anisotropically conductive $\mathrm{E}$ layer induces a non-propagating (evanescent) compressional mode with components $B_{\|}$and $E_{\mathrm{y}}$. The Doppler velocity $V_{2}$ to an incident Alfvén wave was estimated (Pilipenko et al., 2010) as

$V_{2} \simeq-i \omega \frac{\sinh \left|k_{\mathrm{x}} h\right|}{\left|k_{\mathrm{x}}\right|} \frac{\cos I}{B_{0}} J(S) B_{\mathrm{x}}^{(\mathrm{g})}$.

The integral $J(S)=-\int_{0}^{Z_{\mathrm{R}}} \partial_{\mathrm{Z}} \mu \exp \left(-\left|k_{\mathrm{x}}\right| z\right) d z$ has the following asymptotic behavior, depending on parameter $S=$ $\left|k_{\mathrm{x}}\right| z_{\mathrm{R}}$ :

$J(S)= \begin{cases}1-(2 / 3) S+\ldots & (S \ll 1) \\ \frac{1}{2 S}\left(1+\frac{1}{2 S}+\ldots\right) & (S \gg 1) .\end{cases}$

For a latitudinally large-scale disturbance, $k_{\mathrm{x}} h<1, k_{\mathrm{x}} z_{\mathrm{R}}<$ 1, the relationship (12) simplifies to the following:

$V_{2} \simeq-i \omega h \frac{\cos I}{B_{0}} B_{\mathrm{x}}^{(\mathrm{g})}$.

The order-of-magnitude estimate (Pilipenko et al., 2010) of the ratio between the $V_{3}$ and $V_{2}$ disturbances owing to a transformed evanescent compressional mode produced by Alfvén wave is as follows:

$\frac{\left|V_{3}\right|}{\left|V_{2}\right|}=\frac{\left|k_{\mathrm{x}}\right| a}{\cos I}$.

From this estimate it follows that a main contribution into the Doppler response to a latitudinally large-scale Alfvén wave, $\left|k_{\mathrm{x}}\right| a \ll 1$, is determined by the $V_{2}$ mechanism, that is, $\left|V_{3}\right| /\left|V_{2}\right| \ll 1$.

In a realistic situation an incident magnetospheric wave has a finite scale in the azimuthal direction. Indeed, ground observations showed that typical azimuthal wave number of Pi2 pulsations at mid-latitudes is $m \simeq 3$ (Yumoto et al., 2001). A small, but finite, $k_{\mathrm{y}}=m / L R_{\mathrm{E}}$ (where $L$ is the dimensionless distance from the Earth's surface to the top of a field line and $R_{\mathrm{E}}$ is the Earth's radius) value results in the occurrence of non-vanishing $E_{\mathrm{y}}$ component in the incident Alfvén wave. A simple estimate of this component magnitude can be derived from the following consideration. Because in the Alfvén wave $B_{\|}=0$, it follows that $\partial_{\mathrm{x}} E_{\mathrm{y}}-i k_{\mathrm{y}} E_{\mathrm{x}} \simeq \nabla \times\left.\boldsymbol{E}\right|_{\|}=0$, where $E_{\mathrm{x}}$ is the radial component transverse to $\boldsymbol{B}_{0}$. If the wave has a standing-mode structure in the radial direction with scale $a_{\mathrm{x}}\left(a_{\mathrm{x}}^{-1}=\partial \ln E_{\mathrm{y}}\right)$, then $E_{\mathrm{y}} \simeq i\left(k_{\mathrm{y}} a_{\mathrm{x}}\right) E_{\mathrm{x}}$. For a mode propagating in a radial direction, $E_{\mathrm{y}} \simeq-\left(k_{\mathrm{y}} / k_{\mathrm{x}}\right) E_{\mathrm{x}}$.

The main Alfvén wave component $E_{\mathrm{x}}$ is related to the ground magnetic response by the relation $E_{\mathrm{x}}=$ $B_{\mathrm{x}}^{(\mathrm{g})} \sin I / \mu_{0} \Sigma_{\mathrm{H}}$ (Pilipenko et al., 2012). From this relationship it follows that the effective admittance of the system magnetosphere-thin-ionosphere-atmosphere-ground to Alfvén mode is to be

$X^{(\mathrm{A})}=\frac{B_{\mathrm{x}}^{(\mathrm{g})}}{\mu_{\mathrm{o}} E_{\mathrm{x}}}=\frac{\Sigma_{\mathrm{H}}}{\sin I}$. 
The Doppler response to the $E_{\mathrm{y}}$ component of the incident Alfvén wave with $k_{\mathrm{y}} \neq 0$ can be estimated, under the same assumptions as Eq. (13), if one substitutes

$E_{\mathrm{y}}=i\left(k_{\mathrm{y}} a_{\mathrm{x}}\right) \sin I B_{\mathrm{x}}^{(\mathrm{g})} / \mu_{0} \Sigma_{\mathrm{H}}$

into Eq. (3) as follows:

$V_{2}=i \frac{k_{\mathrm{y}} a_{\mathrm{x}} \sin 2 I}{2 \mu_{0} \Sigma_{\mathrm{H}} B_{0}} B_{\mathrm{x}}^{(\mathrm{g})}$.

The radar and ground magnetic observations showed that the coherency scale in the latitudinal direction at low latitudes is large: $>10^{3} \mathrm{~km}$. Therefore, it may be assumed that $a_{\mathrm{x}} \simeq$ $1.2 \times 10^{3} \mathrm{~km}$. For $L \simeq 3$ and $m=3$, the factor $k_{\mathrm{y}} a_{\mathrm{x}} \simeq 0.2$. The comparison of $V_{2}$ caused by Alfvén waves with $k_{\mathrm{y}}=0$ (Eq. 13) and with $k_{\mathrm{y}} \neq 0$ (Eq. 16) shows that in the nighttime ionosphere $V_{2}\left(k_{\mathrm{y}} \neq 0\right) \gg V_{2}\left(k_{\mathrm{y}}=0\right)$ (for the parameters considered the first value is nearly 2 orders of magnitude larger than the second one).

\section{Analysis of synchronous ionospheric and magnetic observations}

We consider the results of simultaneous observations of coherent Doppler and magnetometer Pi2 signatures at the station Paratunka (PTK) with geographic coordinates 52.94 ${ }^{\circ}$, $158.25^{\circ}, \mathrm{LT}=\mathrm{UT}+10.5 \mathrm{~h}$. Altitude of the sounding radio wave reflection $z_{\mathrm{h}} \sim 300 \mathrm{~km}$. According to the IGRF model (http://omniweb.gsfc.nasa.gov/vitmo), for the period of observations the geomagnetic latitude $\Phi=46.5^{\circ}, L=2.2$, and geomagnetic field at $z_{\mathrm{h}}=300 \mathrm{~km}$ is $B_{o}=44800 \mathrm{nT}, H=$ $18800 \mathrm{nT}, Z=40700 \mathrm{nT}$, and declination $D=-5^{\circ}$. Magnetic field inclination is $I=65^{\circ}$. The ionospheric parameters for the period of observations according to the IRI model are $\Sigma_{\mathrm{H}} \sim 0.2 \mathrm{~S}$. Details of the observational system and examples of recorded events can be found in Yumoto et al. (2009) and Ikeda et al. (2010).

To estimate Pi2 E-W electric field, Ikeda et al. (2010) used the relationship $E_{\mathrm{y}}=V_{\mathrm{z}} H$, where $H$ is the geomagnetic field projection on the ground. Because $H=B_{o} \cos I$, and $Z=B_{o} \sin I$, they actually used the relationship $E_{\mathrm{y}}=$ $V_{\mathrm{z}} B_{o} \cos I$. However, the correct relationship (Eq. 3) is $E_{\mathrm{y}}=$ $V_{\mathrm{z}} B_{o} / \cos I$. For typical $V^{*}=15.1 \mathrm{~m} \mathrm{~s}^{-1}, H=18800 \mathrm{nT}$, and $\cos I \simeq 0.4$, they obtained $E_{\mathrm{y}} \simeq 0.28 \mathrm{mV} \mathrm{m}^{-1}$. At the same time, the correct Eq. (3) for the same $V_{\mathrm{z}}=V^{*}$ gives $E_{\mathrm{y}} \simeq 1.7 \mathrm{mV} \mathrm{m}^{-1}$. The latter value is compatible with typical $\boldsymbol{E}$ field amplitudes of Pi2 signals, $\sim 2 \mathrm{mV} \mathrm{m}^{-1}$, detected by SuperDARN (Super Dual Auroral Radar Network) radars (Gjerloev et al., 2007; Teramoto et al., 2012).

For the correct value of $E_{\mathrm{y}} \simeq 1.7 \mathrm{mV} \mathrm{m}^{-1}$ and corresponding $B_{\mathrm{x}}^{(\mathrm{g})} \simeq 3.6 \mathrm{nT}$, the effective admittance of low-latitude $\mathrm{Pi} 2$ wave with $\omega=0.06 \mathrm{~s}^{-1}$ is $X \simeq 1.7 \mathrm{~S}$. This value is much less than the expected admittance of the fast mode, $X \ll$ $X^{(\mathrm{F})}$. Indeed, for $T=100 \mathrm{~s}$ and $z_{h}=300 \mathrm{~km}$, the apparent
Table 1. Mid-latitude $\left(\Phi \sim 50^{\circ}\right)$ Pi2 observations.

\begin{tabular}{lcccc}
\hline & Doppler & SuperDARN & A-model & FMS-model \\
\hline$V\left[\mathrm{~m} \mathrm{~s}^{-1}\right]$ & 15 & 90 & & \\
$E\left[\mathrm{mV} \mathrm{m}^{-1}\right]$ & 1.7 & 4 & & \\
$B[\mathrm{nT}]$ & 3.6 & 5 & & \\
$X[\mathrm{~S}]$ & 1.7 & 1.0 & 0.2 & 40 \\
\hline
\end{tabular}

impedance for a fast mode should be $\left|X^{(\mathrm{F})}\right| \simeq 42 \mathrm{~S}$, that is, nearly 20 times bigger than the observed one. The typical peak-to-peak amplitudes of the Doppler velocity (Ikeda et al., 2010), SuperDARN line-of-sight velocity (Gjerloev et al., 2007), ground magnetic pulsations, derived wave admittance, and theoretically predicted admittances for Alfvén and FMS modes for $\Sigma_{\mathrm{H}}=0.2 \mathrm{~S}$ and $I=65^{\circ}$ are summarized in Table 1.

At the same time, if we assume that the contribution of an Alfvén mode into the ground magnetic signal is $~ 50 \%$ only, that is, $\sim 2 \mathrm{nT}$, this value is sufficient to induce in the nighttime ionosphere with $\Sigma_{\mathrm{H}} \simeq 0.2 \mathrm{~S}$ the electric field disturbance $E_{\mathrm{y}} \simeq 1.4 \mathrm{mV} \mathrm{m}^{-1}$. This estimate following from Eq. (15) indicates that the Doppler response in the ionosphere is produced by the Alfvén wave component, but not the fastmode component.

We suppose that the concept of Pi2 cavity mode mechanism should be augmented by some important aspect. A fast mode trapped in the plasmasphere between the plasmapause and upper ionosphere is coupled, owing to the plasma/field inhomogeneity with Alfvén mode. This suggestion is in conformity with the results of the gradient method analysis of mid-latitude Pi2, which has revealed the occurrence of weak resonance effects in the pulsation latitudinal structure caused by a partial conversion of fast mode into Alfvén waves (Kurchashov et al., 1987). This coupling is not so strong as to modify noticeably the cavity mode eigenfrequency or ground magnetic response to the cavity oscillations. However, the accompanying Alfvén wave induces a stronger electric field in the nighttime ionosphere than a fast mode does. Due to this feature, both radar (Gjerloev et al., 2007; Teramoto et al., 2012) and Doppler (with account of the above correction) (Ikeda et al., 2010) observations consistently indicate the contribution of an Alfvén mode in the structure of Pi2 waves in the low-latitude ionosphere.

The suggestion of coupled cavity and Alfvén modes as the physical nature of Pi2 waves inside the plasmasphere is to be validated by an advanced modeling of MHD spatial structure in a realistic 2-D model comprising both magnetosphere and upper ionosphere.

\section{Conclusion}

We would like to draw attention to the simple and useful technique to identify the wave mode of a 
magnetic-ionospheric disturbance - the effective admittance, which has been applied in this paper. Based on the relationships from the theory of the MHD wave transmission through the thin sheet ionosphere we have obtained simple-to-use analytical estimates for the Doppler responses on incident ULF waves of different types. The combined Doppler-magnetometer observations and theoretical consideration show that Pi2 impulses are produced by coupled fast and Alfvén modes, and the Alfvénic part, relatively weak elsewhere, produces the main contribution into the Pi2induced ionospheric Doppler velocity.

Acknowledgements. This study is supported by the fellowship from STEL, Nagoya University (VP), and program 22 of the Russian Academy of Sciences (EF). We appreciate the thorough reviewing by both referees and the help of K. Ikeda in data analysis.

Topical Editor I. A. Daglis thanks G. Balasis and one anonymous referee for their help in evaluating this paper.

\section{References}

Alperovich, L. S. and Fedorov, E. N.: Hydromagnetic Waves in the Magnetosphere and the Ionosphere, Series: Astrophysics and Space Science Library, 353, XXIV, 418 pp., 2007.

Alperovich, L. S., Fedorov, E. N., Volgin, A. V., Pokhil'ko, S. V., Leus, L. A., Pilipenko, V. A., and Karpov, P. N.: Doppler sounding as tool for the study of MHD wave structure in the ionosphere, J. Atmos. Terr. Phys., 53, 581-586, 1991.

Chum, J., Hruska, F., Buresova, D., Sindelarova, T., Hejda, P., and Bochnice, J.: Ionospheric oscillations caused by geomagnetic $\mathrm{Pi} 2$ pulsations and their observations by multipoint continuous Doppler sounding: First results, Adv. Space Res., 44, 667-676, 2009.

Gjerloev, J. W., Greenwald, R. A., Waters, C. L., Takahashi, K., Sibeck, D., Oksavik, K., Barnes, R., Baker, J., and Ruohoniemi, J. M.: Observations of Pi2 pulsations by the Wallops HF radar in association with substorm expansion, Geophys. Res. Lett., 34, L20103, doi:10.1029/2007GL030492, 2007.

Grant, J. F. and Cole, K. D.: The height dependence of the perturbation of the mid-latitude F-region by Pi2 pulsations, Planet. Space Sci., 40, 1461-1477, 1992.

Han, D. S., Iyemori, T., Nose, M., McCreadie, H., Gao, Y., Yang, F., Yamashita, S., and Stauning, P.: A comparative analysis of lowlatitude $\mathrm{Pi} 2$ pulsations observed by Oersted and ground stations, J. Geophys. Res., 109, A10209, doi:10.1029/2004JA010576, 2004.

Ikeda, A., Yumoto, K., Uozumi, T., Shinohara, M., Nozaki, K., Yoshikawa, A., Bychkov, V. V., and Shevtsov, B. M.: Phase relation between Pi2-associated ionospheric Doppler velocity and magnetic pulsations observed at a midlatitude MAGDAS station, J. Geophys. Res., 115, A02215, doi:10.1029/2009JA014397, 2010.

Keiling, A. and Takahashi, K.: Review of Pi2 models, Space Sci. Rev., 161, 63-148, doi:10.1007/s11214-011-9818-4, 2011.

Kurchashov, Yu. P., Nikomarov, Ya. S., Pilipenko, V. A., and Best, A.: Field-line resonance effects in a local meridional structure of mid-latitude geomagnetic pulsations, Ann. Geophys., 5A, 147154, 1987.

Marshall, R. A. and Menk, F. W.: Observations of Pc 3-4 and Pi 2 geomagnetic pulsations in the low-latitude ionosphere, Ann. Geophys., 17, 1397-1410, doi:10.1007/s00585-999-13972, 1999.

Menk, F. W.: Characterization of ionospheric Doppler oscillations in the Pc3-4 and Pi2 magnetic pulsation frequency range, Planet. Space Sci., 40, 459-507, 1992.

Menk, F. W., Waters, C. L., and Dunlop, I. S.: ULF Doppler oscillations in the low latitude ionosphere, Geophys. Res. Lett., 34, L10104, doi:10.1029/2007GL029300, 2007.

Nishida, A.: Geomagnetic diagnostic of the magnetosphere, Springer-Verlag, New York - Heidelberg - Berlin, 255 p., 1978.

Nose, M.: Excitation mechanism of low-latitude Pi2 pulsations: Cavity mode resonance or BBF-driven process?, J. Geophys. Res., 115, A07221, doi:10.1029/2009JA015205, 2010.

Pilipenko, V., Fedorov, E., Yumoto, K., Ikeda, A., and Sun, T. R.: An analytical model for Doppler frequency variations of ionospheric HF sounding caused by SSC, J. Geophys. Res., 115, A10228, doi:10.1029/2010JA015403, 2010.

Pilipenko, V., Belakhovsky, V., Kozlovsky, A., Fedorov, E., and Kauristie, K.: Determination of the wave mode contribution into the ULF pulsations from combined radar and magnetometer data: Method of apparent impedance, J. Atmos. Solar-Terr. Phys., 77, 85-95, doi:10.1016/j.jastp.2011.11.013, 2012.

Pilipenko, V., Fedorov, E., Heilig, B., Engebretson, M. J., Sutcliffe, P., and Luehr, H.: ULF waves in the topside ionosphere: Satellite observations and modeling, in: The Dynamic Magnetosphere, IAGA Special Sopron Book Series, vol. 3, edited by: Liu, W. and Fujimoto, M., Chapter 14, Springer, 257-269, doi:10.1007/97894-007-0501-2, 2012.

Poole, A. W. V., Sutcliffe, P. R., and Walker, A. D. M.: The relationship between ULF geomagnetic pulsations and ionospheric Doppler oscillations: Derivation of a model, J. Geophys. Res., 93, 14656-14664, 1988.

Reddy, C. A., Ravindran, Sudha, Viswanathan, K. S., Krishna Murthy, B. V., Rao, D. R. K., and Araki, T.: Observations of Pc5 micropulsation-related electric field oscillations in the equatorial ionosphere, Ann. Geophys., 12, 565-573, doi:10.1007/s00585994-0565-7, 1994.

Schunk, R. W. and Nagy, A. F.: Ionospheres: Physics, Plasma Physics, and Chemistry, Cambridge University Press, 2009.

Shiokawa, K., Baumjohann, W., Haerendel, G., Paschmann, G., Fennell, J. F., Friis-Christensen, E., Lühr, H., Reeves, G. D., Russell, C. T., Sutcliffe, P. R., and Takahashi, K.: High-speed ion flow, substorm current wedge, and multiple $\mathrm{Pi} 2$ pulsations, J. Geophys. Res., 103, 4491-4508, doi:10.1029/97JA01680, 1998.

Sutcliffe, P. R. and Luhr, H.: A comparison of Pi2 pulsations observed by CHAMP in low Earth orbit and on the ground at low latitudes, Geophys. Res. Lett., 30, 2105, doi:10.1029/2003GL018270, 2003.

Sutcliffe, P. R. and Poole, A. W. V.: Low latitude Pc3 pulsations and associated ionospheric oscillations measured by a digital chirp ionosonde, Geophys. Res. Lett., 11, 1172-1175, 1984.

Sutcliffe, P. R. and Poole, A. W. V.: Ionospheric Doppler and electron velocities in the presence of ULF waves, J. Geophys. Res., 94, 13505-13514, 1989. 
Sutcliffe, P. R. and Poole, A. W. V.: The relationship between ULF geomagnetic pulsations and ionospheric Doppler oscillations: model predictions, Planet. Space Science, 38, 1581-1589, 1990.

Sutcliffe, P. R. and Yumoto, K.: On the cavity mode nature of lowlatitude Pi2 pulsations, J. Geophys. Res., 96, 1543-1551, 1991.

Takahashi, K., Ohtani, S.-I., and Anderson, B. J.: Statistical analysis of $\mathrm{Pi} 2$ pulsation observed by the AMPTE CCE spacecraft in the inner magnetosphere, J. Geophys. Res., 100, 21929-21941, 1995.

Takahashi, K., Ohtani, S., Hughes, W. J., and Anderson, R. R.: CRRES satellite observations associated with low-latitude Pi2 pulsations, J. Geophys. Res., 106, 15567-15581, 2001.

Takahashi, K., Lee, D.-H., Nose, M., Anderson, R. R., and Hughes, W. J.: CRRES electric field study of the radial mode structure of Pi2 pulsations, J. Geophys. Res., 108, 1210, doi:10.1029/2002JA009761, 2003.

Teramoto, M., Nishitani, N., Ogawa, T., Shiokawa, K., Nagatsuma, T., and Murata, K. T.: Spatial characteristics of Pi2 pulsations in the ionosphere observed by the SuperDARN Hokkaido radar, GEMSIS International Workshop, 12-14 March 2012, Nagoya University, Japan, 2012.
Wright, D. M., Yeoman, T. K., and Chapman, P. J.: High-latitude HF Doppler observations of ULF waves. 1. Waves with large spatial scale sizes, Ann. Geophys., 15, 1548-1556, doi:10.1007/s00585997-1548-2, 1997.

Yeoman, T. K. and Orr, D.: Phase and spectral power of mid-latitude Pi2 pulsations: Evidence for a plasmaspheric cavity resonance, Planet. Space Sci., 38, 1367-1383, 1989.

Yumoto, K. and the CPMN Group: Characteristics of Pi2 magnetic pulsations observed at the CPMN stations: A review of the STEP results, Earth Planets Space, 53, 981-992, 2001.

Yumoto, K., Ikeda, A., Shinohara, M., Uozumi, T., Nozaki, K., Watari, S., Kitamura, K., Bychkov, V. V., and Shevtsov, B. M.: Electric and magnetic field variations at low and equatorial latitudes during SC, DP2, and Pi2 Events, Adv. Geosci., 14, Solar Terrestrial, edited by: Duldig, M., World Sci. Publ. Comp., 197212, 2009. 